\title{
Sociedad Mexicana de Neumología y Cirugía de Tórax, A.C. Informe de Labores de la Mesa Directiva 2015-2017
}

\author{
Juan Carlos Vázquez-García $\bowtie$ \\ Presidente de la Sociedad Mexicana de Neumología y Cirugía de Tórax, A.C., 2015-2017; \\ Director de Enseñanza, Instituto Nacional de Enfermedades Respiratorias Ismael Cosío Villegas, Ciudad de México. \\ Trabajo recibido: 17-V-2017; aceptado: 17-V-2017
}

Durante el pasado Congreso Anual de la Sociedad Mexicana de Neumología y Cirugía de Tórax, A.C. (SMNyCT), 76 Congreso de las Américas, celebrado en la ciudad de Puebla, Puebla, del 17 al 21 de abril de 2017, de acuerdo con los estatutos que rigen a la sociedad, se presentó ante la Asamblea General el informe final de la mesa directiva 2015-2017 y que fuera acorde a la propuesta original de trabajo. El informe final fue aprobado en el pleno de la asamblea y se puede detallar en los siguientes logros:

\section{FINANCIAMIENTO RÉCORD CON UN EJERCI- CIO TRANSPARENTE DEL PRESUPUESTO}

El Dr. Enrique Guzmán de Alba, tesorero de la Sociedad, presentó a la Asamblea un informe detallado del presupuesto que inició con los fondos finales correspondientes al cierre final de la mesa directiva anterior, el financiamiento obtenido durante los dos años de ejercicio y que corresponden a las cuotas de los socios, a ingresos específicos por ganancias de los dos congresos celebrados, los patrocinios específicos obtenidos para rubros de educación continua, investigación y apoyo a programas de salud respiratoria. Estos últimos por sus características (especificadas más adelante) y los montos obtenidos son de relevancia histórica. Además, el presupuesto en sus ingresos y egresos se ejerció de forma planeada, equilibrada y transparente, con la finalidad de recuperar la salud financiera de la sociedad, lo que le permitió alcanzar sus objetivos de educación, investigación y promoción de la salud respiratoria.

\section{SE ORGANIZARON DOS CONGRESOS DE LA MAYOR CALIDAD CIENTÍFICA INTERNACIONAL}

Un objetivo mayor de la mesa directiva fue organizar los congresos anuales de la Sociedad con la mayor calidad académica, científica y social que, además, representaran un éxito económico. En la tabla 1 se resumen los principales indicadores de los dos congresos organizados, el 75 Congreso Diamante realizado en la Ciudad de Guadalajara del 28 de marzo al $1^{\circ}$ de abril de 2016, así como el 76 Congreso de las Américas en la Ciudad de Puebla del 17 al 21 de abril de 2017. Aun cuando el Congreso Diamante se consideró exitoso, particularmente por ser un congreso de aniversario, fue superado significativamente por el congreso 76 de las Américas, el cual estableció récords históricos en la mayoría de sus indicadores. Sin duda, el congreso de nuestra Sociedad se ha consolidado como un evento de calidad y referencia internacional que incluye foros multidisciplinarios de neumología, neumología pediátrica, cirugía de tórax, así como enfermería y terapia respiratoria.

\section{MAYOR INTEGRACIÓN Y COLABORACIÓN CON SOCIEDADES HERMANAS NACIONALES E INTERNACIONALES}

La SMNyCT estableció una política de consolidación, acercamiento y colaboración con asociaciones similares de lucha común de carácter nacional o internacional. Los principales convenios de colaboración internacional de la sociedad son:

I. Se firmó un convenio de colaboración con la Asociación Latinoamericana del Tórax (ALAT) y la Sociedad Europea Respiratoria (ERS) que incluye una triple membresía con un pago único, el cual no significó un incremento significativo en las cuotas anuales de los socios; además, incluye el intercambio de profesores y la organización de actividades académicas o científicas conjuntas. 
II. Se mantuvo el convenio de colaboración con la Sociedad Española de Neumología y Cirugía Torácica (SEPAR) que incluye el intercambio de profesores y una beca de estancia corta (Beca SMNyCT-SEPAR); además, se acordó la organización de simposios y actividades científicas institucionales.

III. Se ratificó el convenio de colaboración con la Asociación Argentina de Medicina Respiratoria (AAMR) que incluye el intercambio de profesores y se amplía a una beca de estancia corta (Beca SMNyCTAAMR); además, se acordó la organización de simposios y actividades científicas institucionales.

IV. Se firmó un convenio de colaboración con la Federación Centroamericana y del Caribe de Neumología y Cirugía de Tórax (FCCNCT) que incluye el intercambio de profesores y actividades académicas o científicas comunes en sus congresos, así como membresía asociada de la SMNyCT otorgada a los miembros activos de la Federación.

V. Finalmente, durante el pasado 76 Congreso se firmó un convenio de colaboración con la Sociedad de Enfermedades Respiratorias de Chile (SERChile) que incluye el intercambio de profesores y la organización de actividades científicas comunes.

\section{UNA SMNYCT MÁS INSTITUCIONAL}

El crecimiento natural de la Sociedad, tanto en el número de socios como en sus diversas actividades, obliga a que cuente con una estructura organizacional más amplia y eficiente, aunque más compleja. Durante la Asamblea General legalmente instalada el 31 de marzo de 2016, el Presidente de la SMNyCT propuso una serie de reformas estatutarias que fueron debidamente aprobadas y representan un cambio mayor en la institucionalización de la sociedad. Estas reformas incluyen los siguientes cambios:

I. La creación de un Comité Ejecutivo compuesto por el Presidente, Vicepresidente, Presidente Pasado, Secretario, Tesorero, Jefes de las Divisiones de Neumología, Neumopediatría y Cirugía de Tórax, así como la integración del Comisario.

II. Se crea la figura de un Comisario Fiscal quien fue elegido directamente por la Asamblea (Dr. José Felipe Villegas Elizondo, expresidente de la Sociedad) y con una duración indefinida. El Comisario es responsable de la aprobación anual de las cuentas y estados financieros de la Sociedad.

III. Se aprueba la creación de un Comité de Investigación y Educación que deberá estar conformado por cinco socios de prestigio reconocido en el contexto académico profesional y de investigación científica. El Comité será responsable de la gestión, promoción y operación de las actividades propias de educación continua y de investigación que deberá promocionar la Asociación.

IV. Se aprueba la creación de grupos científicos o Departamentos de la División de Neumología. Los socios neumólogos podrán organizarse en grupos científicos

Tabla 1. Numeraria de los Congresos de las SMNyCT 2016 y 2017.

\begin{tabular}{lrr}
\hline & 2016 & 2017 \\
& 75 Congreso Diamante & 76 Congreso de las Américas \\
\hline Asistentes & 1,168 & 1,630 \\
Trabajos libres & 178 & 253 \\
Conferencias & 290 & 482 \\
Profesores extranjeros & 30 & 53 \\
Profesores nacionales & 128 & 242 \\
Cursos para posgraduados & 2 & 4 \\
Talleres especializados & 6 & 8 \\
Residentes becados & 60 & 60 \\
Curso nacional de TB & 130 & 150 \\
Congreso de enfermería* & 77 & 80 \\
Congreso de TR* & 50 & 59 \\
Simposios institucionales & 3 & 3 \\
Simposios Diamante & 8 & 4 \\
Desayunos con el experto & 2 & 2 \\
Comidas con el experto & - & 1 \\
Expositores comerciales & 27 & 32 \\
Expositores no comerciales & 6 & 5 \\
\hline
\end{tabular}

*Asistentes al congreso de Enfermería y al de Terapia Respiratoria (TR). 
que serán aprobados por el Comité Ejecutivo. Estos grupos podrán elegir democráticamente un Jefe de Departamento y deberán proponer un plan de trabajo que incluya las actividades propias de los congresos, de educación continua, de investigación, generación de normativas y recomendaciones, así como promoción de la salud propia de la materia.

V. Se aprueba una mayor autonomía y democracia de los capítulos estatales. La sociedad reconoce oficialmente a las sociedades estatales y a sus representantes como gobernadores de los capítulos de las entidades federativas. Sin embargo, en caso de que los estados no cuenten con una asociación propia, la SMNyCT reconocerá a los representantes (un Gobernador del Capítulo y un Secretario) democráticamente electos por mayoría de votos de los socios activos registrados en la entidad federativa correspondiente.

\section{CUERPO DE GOBIERNO CORPORATIVO Y PROFESIONAL}

Las metas establecidas en el programa bianual de trabajo requirieron de una reingeniería profunda de la organización de la SMNyCT. Además de la mesa directiva, por primera vez se integró un equipo de trabajo profesional compuesto principalmente por un Director Ejecutivo responsable de la coordinación general del personal y de las actividades de la sociedad y una Dirección Administrativa a cargo del Buffet J. Hoffman Consultoras, así como el personal administrativo conformado por tres asistentes ejecutivas y una contadora pública especializada, cada una con funciones claras y con procesos establecidos y supervisados para las actividades propias de la Sociedad. Además, es recomendable que en un futuro cercano la Sociedad cuente con una Dirección de Mercadotecnia que le permita incrementar y coordinar mejor los patrocinios de sus congresos y de todas sus actividades susceptibles de financiamiento. La reestructuración de la Sociedad conjuntamente con la creación de un Comité Ejecutivo (reforma estatutaria 2016) consolidará un cuerpo de gobierno corporativo a la altura de sus objetivos.

\section{CRECIMIENTO DE LA MEMBRESÍA ACTIVA}

Una obligación mayor de los miembros de la SMNyCT es mantenerse al corriente del pago de su membresía anual. Sin embargo, en el primer cuatrimestre de 2015 sólo existían 220 socios activos al corriente de sus obligaciones de los poco más de mil registrados. Durante este bienio, se estableció una política de regularización de pagos de membresía que para ma- yor éxito se acompañó de una política de beneficios al socio. Actualmente los socios al corriente de su membresía son sujetos de múltiples beneficios que incluyen: la triple membresía (SMNyCT-ALAT-ERS), un costo de congreso significativamente mejor, acceso a múltiples actividades de educación continua con becas parciales o completas y seguir recibiendo un ejemplar de la revista NCT por correspondencia. Al final de la administración el número de socios activos se incrementó a más de 700 .

\section{FORTALECIMIENTO DE LAS DIVISIONES Y SECCIONES CIENTÍFICAS}

En la estructura orgánica de la Sociedad las Divisiones numerarias compuestas por neumología, neumopediatría y cirugía de tórax son pilares fundamentales. El apoyo a las Divisiones fue irrestricto, particularmente para la organización de sus congresos. Es importante mencionar que durante la gestión se reinstalaron las actividades de la División de Enfermería y Terapia Respiratoria, creada estatutariamente; nuestros congresos 2015 y 2016 incluyeron sus congresos de enfermería y terapia respiratoria con gran éxito. Asimismo, durante esta gestión y con la finalidad de organizar y fortalecer las actividades académicas de la división de neumología se confirmaron 11 secciones científicas a cargo de 23 reconocidos miembros de la Sociedad, quienes junto a los Jefes de División fueron invaluables para la organización de los congresos, a todos ellos nuestro mayor reconocimiento. Estamos seguros que gracias a las reformas estatutarias de la sociedad, la mayor parte de las secciones científicas se consolidarán en grupos de trabajo organizados como departamentos científicos que les permitirá desarrollar y concretar proyectos de largo plazo y de mayor alcance.

\section{FORTALECIMIENTO Y AUTONOMÍA DE LOS CAPÍTULOS ESTATALES}

El crecimiento en el número de especialistas respiratorios ha sido una constante en la mayoría de los estados de la República y cada vez se encuentran más organizados y académicamente más activos. Durante la gestión 2015-2017 se reconocieron, promovieron y en algunos casos se coorganizaron eventos académicos en las entidades federativas. Debemos reconocer la exitosa organización del Capítulo Metropolitano de la Ciudad de México, encabezado por la Dra. Lilia Gordon Vásquez, quien organizó de forma ininterrumpida sesiones mensuales con ponencias de alto nivel académico y concurrida asistencia. Finalmente en la próxima administración se hará efectivo el cambio es- 
tatutario, entrará el cambio para que el representante estatal sea democráticamente electo por los socios registrados en la entidad, lo que fortalecerá aún más los capítulos.

\section{DESARROLLO DE UN PROGRAMA DE EDUCA- CIÓN CONTINUA DE LARGO PLAZO}

El crecimiento y evolución vertiginosa del conocimiento médico y de sus avances tecnológicos es una característica inherente a la medicina especializada. Esto obliga a los profesionales médicos a la búsqueda del fortalecimiento curricular; la SMNyCT se ha convertido en la mayor fuente de actualización profesional de las especialidades respiratorias de nuestro país. El programa de educación continua de la gestión fue uno de los mayores objetivos planteados y se enfocó principalmente en el curso MECOR, los cursos para posgraduados y en el Programa de Neumología al Día.

La SMNyCT firmó un convenio de colaboración con la ALAT para la organización de dos cursos MECOR (Methods in Epidemiologic, Clinical and Operations Research) fundado y también auspiciado por la Sociedad Americana del Tórax (ATS). Este prestigioso curso enfocado en el entrenamiento en investigación clínica y epidemiológica en medicina respiratoria fue organizado del 9 al 14 de noviembre de 2015 en la Ciudad de Puebla con la asistencia internacional de 100 alumnos inscritos en sus cuatro niveles académicos, quienes compartieron una intensa jornada científica con 12 profesores investigadores de reconocido prestigio encabezados por la Dra. Ana María Meneses y la Dra. Sonia Buist. Si bien el segundo curso está programado para el año 2019, los especialistas de nuestro país requieren de una actividad de este tipo y calidad con una organización más frecuente, por lo menos anual.

Los mayores requerimientos de educación continua de nuestros especialistas son relacionados al fortalecimiento de competencias para el manejo de las enfer- medades respiratorias. De forma planeada y en acuerdo con el Consejo Nacional de Neumología se desarrolló el programa de actualización por competencias de neumología (PAC Neumo). Este programa consiste en el desarrollo de cursos de educación continua de 16 horas, enfocados y estructurados en el desarrollo de competencias específicas. Estos cursos presenciales y de aprobación obligatoria son reconocidos por el CNN con puntajes significativos para la recertificación de especialistas. En el seno de nuestros congresos se desarrollaron dos cursos de este modelo en 2016 (EPOC y ventilación no invasiva) y tres en 2017 (ventilación no invasiva, asma y fisiología).

Desde hace décadas la SMNyCT organiza de forma anual un curso de Neumología al Día con fines de actualización para la recertificación de nuestros especialistas; durante 2015 y 2016 en acuerdo con el CNN, el Instituto Nacional de Enfermedades Respiratorias (INER) y la Sociedad Médica del Hospital ABC y con reconocimiento de ALAT. Se organizaron las versiones de Neumología al Día y Neumopediatría al Día en un formato de tres y dos días, respectivamente, con conferencias matutinas y talleres prácticos vespertinos. Ambos eventos fueron muy exitosos y sus principales indicadores se resumen en la tabla 2. Cabe mencionar que para la organización de ambos cursos se consiguió el patrocinio de la industria farmacéutica, por lo que los socios activos asistentes fueron becados en su totalidad.

\section{PROGRAMA DE APOYO A LAS SEDES FOR- MADORAS DE ESPECIALISTAS}

Desde el año 2010 la SMNyCT ha auspiciados reuniones periódicas de los profesores de neumología. Los días 5 y 6 de agosto de 2016, en la Ciudad de Cuernavaca se organizó una reunión con todos los profesores de neumología, neumopediatría y cirugía de tórax, así como la presencia oficial del CNN y con el objetivo del fortalecimiento de las sedes formadoras de nuestros

Tabla 2. Neumología al Día 2015 y 2016.

\begin{tabular}{lccccc}
\hline & \multicolumn{2}{c}{ Neumología al Día 2015 } & & \multicolumn{2}{c}{ Neumología al Día 2016 } \\
\cline { 2 - 3 } \cline { 5 - 6 } & Neumología & Neumopediatrían & & Neumología & Neumopediatría \\
\hline Asistentes & 157 & 100 & 150 & 160 \\
Conferencias & 35 & 1 & & 28 & 17 \\
Talleres & 4 & - & 3 & 3 \\
Profesores extranjeros & 4 & 17 & 22 & - \\
Profesores nacionales & 31 & &
\end{tabular}


especialistas. Este grupo destacado de la sociedad, junto con otros reconocidos académicos, deberá ser fortalecido en los próximos años con la finalidad de consensar los programas académicos de las especialidades, así como los de educación continua.

Otro logro mayor que hay que destacar es la organización muy exitosa del Foro de Residentes durante nuestros congresos 2016 y 2017. Durante un día completo, en cada congreso se reunieron casi la totalidad de los residentes del último año de nuestras especialidades, provenientes de todas las sedes académicas, quienes intercambiaron conocimientos y experiencias con seis profesores nacionales y seis profesores extranjeros. Este evento fue posible por las becas otorgadas por parte de la industria farmacéutica para la totalidad de los médicos residentes.

\section{DESARROLLO DE NORMATIVAS Y GUÍAS DE PRÁCTICA PROFESIONAL (GUIMA 2017)}

Un deber mayor de las asociaciones médicas profesionales es el auspicio para el desarrollo de normativas, recomendaciones y guías de práctica profesional. En los próximos años ésta deberá ser una responsabilidad mayor de los grupos de trabajo de nuestra sociedad. Un ejemplo a seguir fue el desarrollo de la Guía Mexicana de Asma (GUIMA 2017). Por gestión de la SMNyCT y convocatoria conjunta con el INER se integró un grupo de trabajo de 58 expertos y participantes oficiales de 11 sociedades profesionales de neumología, alergología, otorrinolaringología, pediatría, medicina general y familiar; además de instituciones como el INER, el Instituto Nacional de Pediatría, el Hospital Infantil de México y el Centro Nacional de Programas Preventivos y Control de Enfermedades (CENAPRECE) de la Secretaría de Salud. Gracias al patrocinio completo de la industria farmacéutica, gestionado y administrado por la SMNyCT y después de 18 meses de trabajo y tres reuniones presenciales, se presentó en nuestro pasado congreso GUIMA 2017 como una publicación simultánea en números especiales de las revistas NCT y la Revista Alergia México.

\section{ALIANZA CONTRA LAS ENFERMEDADES RESPIRATORIAS (PROGRAMA RENACE)}

Sin duda la atención y el control de las enfermedades respiratorias agudas y crónicas, que en su conjunto son uno de los mayores problemas de salud pública en México y en el mundo, son un reto mayor para nuestras especialidades. Sólo una alianza de todos los profesionales, las instituciones y las organizaciones no gubernamentales como la SMNyCT, pueden hacer un frente común a la altura de este gran reto. Este objetivo planteado en el programa bianual de trabajo tuvo avances mayores y muy alentadores. La SMNyCT gestionó y logró un patrocinio mayor sin restricciones de la empresa GSK para la implementación de un programa de diagnóstico de enfermedades respiratorias en primero y segundo nivel de atención. En el año 2015 se firmó un convenio de colaboración entre la SMNyCT, el INER y GSK para la capacitación del personal de centros de atención primaria y secundaria y la implementación de una Red Nacional de Espirometría (RENACE). Este financiamiento incluyó el donativo de 40 espirómetros, 100 oxímetros de pulso y recursos económicos para el entrenamiento y la implementación de los centros de diagnóstico. A la fecha se han implementado más de 28 centros y capacitado más de 200 técnicos en espirometría que ya han realizado más de 1,400 espirometrías en ocho estados de la república. Este proyecto será un programa multiinstitucional (SMNyCT-INER-Secretaría de Salud) de largo plazo; y la SMNyCT seguirá siendo la responsable de la gestión y administración de los donativos de forma auditable y transparente que esperamos se extienda por parte de toda la industria farmacéutica.

\section{GESTIÓN Y PROMOCIÓN DE INVESTIGACIÓN CIENTÍFICA}

La gestión y la promoción de investigación científica es uno de los mayores alcances de las sociedades médicas profesionales pero que, desafortunadamente, no ha sido desarrollado por completo en la SMNyCT. Este objetivo planteado de manera inicial en el programa de trabajo concluyó en una propuesta de un proyecto mayor de investigación epidemiológica: Proyecto Mexicano de Salud Respiratoria (estudio PROMESA). El proyecto diseñado como un estudio de base poblacional para la medición de la prevalencia de las principales enfermedades respiratorias y sus fenotipos (asma, EPOC y apnea del sueño) en tres ciudades representativas de la población mexicana no pudo concretar su financiamiento. Ahora, será responsabilidad del naciente Comité de Investigación y Educación así como de la nueva mesa directiva, gestionar y administrar el desarrollo de este estudio y otros más, lo cuales nos destacarán y nos ayudarán a contribuir con un mejor conocimiento científico de calidad mundial de los problemas de salud respiratoria de nuestro país.

\section{FORTALECIMIENTO DE NCT}

La revista NCT, al igual que la SMNyCT, está cercana a cumplir sus primeros 80 años de vida. Durante la pasada Asamblea General el Dr. Patricio Santillán Doherty, 
Editor en Jefe, presentó un informe oficial con detalles de su desarrollo y crecimiento. Si bien NCT mantiene su publicación regular y cada vez de mayor calidad científica, requiere de un plan estratégico de inversión que asegure su crecimiento y desarrollo para convertirla en un referente de la materia y de mayor competencia internacional.

\section{UNA SMNYCT CERCANA AL SOCIO}

La SMNyCT pertenece a todos y cada uno de sus asociados, por lo que se implementó un programa de acercamiento permanente y continuo con sus miembros. La generación de un Newsletter, publicada en 12 números durante la gestión, así como su página Web y sus redes sociales fueron implementadas y/o actualizadas con un exitoso crecimiento, lo que permitió tener los mejores canales de comunicación con todos y cada uno de sus miembros.

Mesa Directiva de la SMNYCT 2015-2017

\section{Dr. Juan Carlos Vázquez García}

Presidente
Dra. Mayra Edith Mejía Ávila

Vicepresidente

Dr. Jorge Luis Ramírez Figueroa

Secretario

\section{Dr. Enrique Guzmán de Alba}

Tesorero

La Mesa Directiva de la SMNyCT agradece profundamente la confianza otorgada por sus socios y a todo el staff de la Sociedad por su trabajo, entrega y sobre todo el gran entusiasmo y cariño que mostraron a nuestra asociación.

$\triangle$ Correspondencia:

Dr. Juan Carlos Vázquez-García,

Dirección de Enseñanza, Instituto Nacional de

Enfermedades Respiratorias Ismael Cosío Villegas,

Calzada de Tlalpan Núm. 4502

Colonia Sección XVI, 14080,

Del. Tlalpan, Ciudad de México.

Teléfono: 555487 1743, ext. 5146

Correo electrónico: drjcvazquez@gmail.com

El autor declara no tener conflicto de intereses. 